\title{
Lead Device
}

National Cancer Institute

\section{Source}

National Cancer Institute. Lead Device. NCI Thesaurus. Code C50282.

An insulated electrical conductor designed to connect to an electrical device. 